\title{
Specified Influence of Painful Diabetic Neuropathy on Quality of Life in Egyptian Patients with Type 2 Diabetes Mellitus \\ Sally Sameh Ahmed Mohammed ${ }^{1}$, Omnia Ibrahim Metwally State ${ }^{1}$, Ahmed Hassan Elsebaie ${ }^{2}$, Mohammed Ali Gameil ${ }^{1 *}$ \\ Departments of ${ }^{1}$ Internal medicine and ${ }^{2}$ Clinical Pathology, Faculty of Medicine, Mansoura University. Corresponding Author: Mohammed Ali Gameil, Mobile: 00201099975071 Email: drmaligameil1979@ yahoo.com
}

\begin{abstract}
Background: There is a lack of data in discrimination of different aspects of quality of life impairment in Egyptian patients with painful diabetic neuropathy (PDN).

Objective: To assess the influence of PDN on different aspects of life quality in patients with type 2 diabetes mellitus (T2D).

Patients and methods: A cross-sectional study that included 125 patients with T2D (100 with PDN and 25 without) during the period from December 2018 to May 2019. All patients were subjected to history, examination with modified Neuropathy Disability Score (NDS), neuropathy symptom score (NSS), visual analogue pain score (VAS), nerve conduction study for (peroneal, sural and ulnar nerves) bilaterally and Norfolk Quality of Life QuestionnaireDiabetic Neuropathy (Norfolk QOL-DN) and lab. Other neuropathy causes were excluded.

Results: PDN patients have longer duration of T2D. Norfolk QOL-DN showed a significant deterioration of the activity of daily life (ADL) and general health status in PDN patients. We found a significant positive correlation between the NSS, VAS, NDS, superficial pain sensation, and the duration of T2D with the effects on ADL and health status in the case group. In addition, there was a significant positive correlation between insulin therapy, HbA1c, electrical sensation, NSS, VAS, NDS and the duration of T2D with axonal neuropathy. The NDS was the only independent predictor of ADL and health status impairment. Axonal neuropathy was independently determined by NDS, electrical sensation and insulin therapy.

Conclusion: PDN impaired the activity of daily life and health status of patients with T2D. The NDS independently could predict axonal neuropathy, ADL and health status impairment.

Keywords: Quality of life, Painful diabetic neuropathy, Norfolk Quality of Life Questionnaire-Diabetic Neuropathy (Norfolk QOL-DN).
\end{abstract}

\section{INTRODUCTION}

Diabetic neuropathy is identified by the presence of peripheral nerve dysfunction in diabetic patients with exclusion of other causes (1). The mechanisms involved in the development of neurological manifestations of diabetes are complex and poorly understood ${ }^{(2,3)}$. Metabolic abnormalities in diabetic patients have a principal role in painful diabetic neuropathy (PDN) development. Oxidative stress, advanced glycation end product, activation of the polyol pathway, increased sorbitol and fructose deposition, myo-inositol depletion, $\mathrm{Na}^{+} /$ATPase inhibition, neurotropism deficiency with depletion of neurotrophic factors and deranged axonal transport are the main pathological features of diabetic peripheral neuropathy (4, 5, 6). Worldwide, PDN represents a clinical challenge. Diabetic patients may have hyperalgesia, allodynia, hypothesis and spontaneous pain ${ }^{(7,8)}$.

PDN has a substantial effect on the diabetic patients' life. It interferes with the patient's selfmanagement through decreased activity and loss of motivation with increased risk of fall down and fractures due to movement incoordination and weakness ${ }^{(9)}$.
The Norfolk Quality of Life QuestionnaireDiabetic Neuropathy (Norfolk QOL-DN) is considered as an integrated model that covers the whole spectrum of diabetic neuropathy ${ }^{(10)}$. Our aim was to get a delicate discrimination of various aspects of QOL impairment induced by PDN in Egyptian patients with type 2 diabetes mellitus (T2D).

\section{PATIENTS AND METHODS}

An observational cross sectional study that included 125 patients with T2D (100 with diabetic neuropathy and 25 without neuropathy) and was conducted at the Outpatient Clinics of our hospital during the period from December 2018 to May 2019. Detailed medical history (age, sex, duration of diabetes, therapeutic history, smoking and presence of hypertension or diabetic foot lesion). Clinical examination with anthropometric measures (height, body weight and BMI). Laboratory assessment (fasting and postprandial plasma glucose, HBA1c, lipid profile, TSH, electrolytes, hepatic and renal functions and vitamins $(25(\mathrm{OH})$ vitamin $\mathrm{D}$ and $\mathrm{B} 12)$ were done for each participant

Exclusion criteria: We excluded patients with endocrine disorders (Thyroid disease, acromegaly, 
etc.), prior amputation, connective tissue disease, nutritional disorders, malignancy, liver cell failure, renal failure, heart failure, COPD, neuropsychiatric disorders, other causes of neuropathy (e.g. radiculopathy) and patients receiving neurotoxic drugs (e.g. isoniazid).

\section{Ethical Approval:}

Written informed consent was obtained from all participants prior to enrolment. Our study got approval from the Institutional Review Board (IRB) for Clinical Research Committee of our university.

Diagnosis of peripheral neuropathy was established by the following tools:

I. Neuropathy symptom score (NSS), which is consisted of 5 questions with total score of 9 (3-4 points $=$ mild, $5-6$ points $=$ moderate and 7-9 points $=$ severe $)^{(11)}$.

II. The Neuropathy Disability Score (NDS), which is composed of four clinical tests on both feet. (Vibration perception by $128 \mathrm{~Hz}$ tuning fork, temperature perception, ankle reflex and pinprick test) with $10 \mathrm{~g}$-SemmesWeinstein Monofilament for pressure sensation $^{(12)}$.
III Nerve conduction study was done bilaterally for peroneal (motor), sural (sensory) and ulnar (motor and sensory) nerves using standard techniques in the electrophysiology lab. The mean of the action potential amplitude, distal latency and conduction velocity of each nerve were recorded.

IV. Visual analogue pain score (VAS) for evaluation of pain severity ${ }^{(\mathbf{1 3})}$.

V. The Norfolk Quality of life QuestionnaireDiabetic Neuropathy (Norfolk QOL-DN) ${ }^{(10)}$.

\section{Statistical Analysis}

Analysis was carried out using Statistical Program for Social Science (statistical package) version 17 (SPSS Inc., Chicago, IL, USA). Some statistics such as frequency, arithmetic mean and standard deviation were used. Student's t-test and the Mann-Whitney U-test were used for the parametric data and nonparametric data respectively. Linear correlations were performed by Pearson's test. Stepwise logistic regression was used for multivariable analysis. For all analyses $\mathrm{P} \leq 0.05$ indicates statistical significance.

\section{RESULTS}

Table (1): Demographic clinical data of studied patients.

\begin{tabular}{|l|c|c|c|}
\hline Characteristics & $\begin{array}{c}\text { Cases } \\
\text { No .(100) }\end{array}$ & $\begin{array}{c}\text { Control } \\
\text { No.(25) }\end{array}$ & P-value \\
\hline Age (years) & $47.5 \pm 9.9$ & $43.9 \pm 8.9$ & 0.102 \\
\hline $\begin{array}{l}\text { Gender (No. \%) Male } \\
\text { Female }\end{array}$ & $\begin{array}{c}53(53) \\
47(47)\end{array}$ & $\begin{array}{l}11(44) \\
14(56)\end{array}$ & 0.421 \\
\hline $\begin{array}{l}\text { Medication (No. \%) } \\
\text { Oral antidiabetics } \\
\text { Insulin }\end{array}$ & $36(36)$ & $15(60)$ & $0.029^{*}$ \\
\hline BMI (Kg/m $)$ & $64(64)$ & $24.3 \pm 1.9$ & 0.202 \\
\hline HbA1C (\%) & $25.1 \pm 3.1$ & $6.9 \pm 0.4$ & 0.524 \\
\hline Duration of DM (years) & $7.0 \pm 0.7$ & $1(0.3-6)$ & $\leq 0.001^{*}$ \\
\hline
\end{tabular}

Table (1) showed the demographic data of the study groups. The duration of diabetes was significantly longer in the case group versus the control group $(\mathrm{P}<0.001)$. 
Table (2): The Norfolk quality of life questionnaire of the study groups.

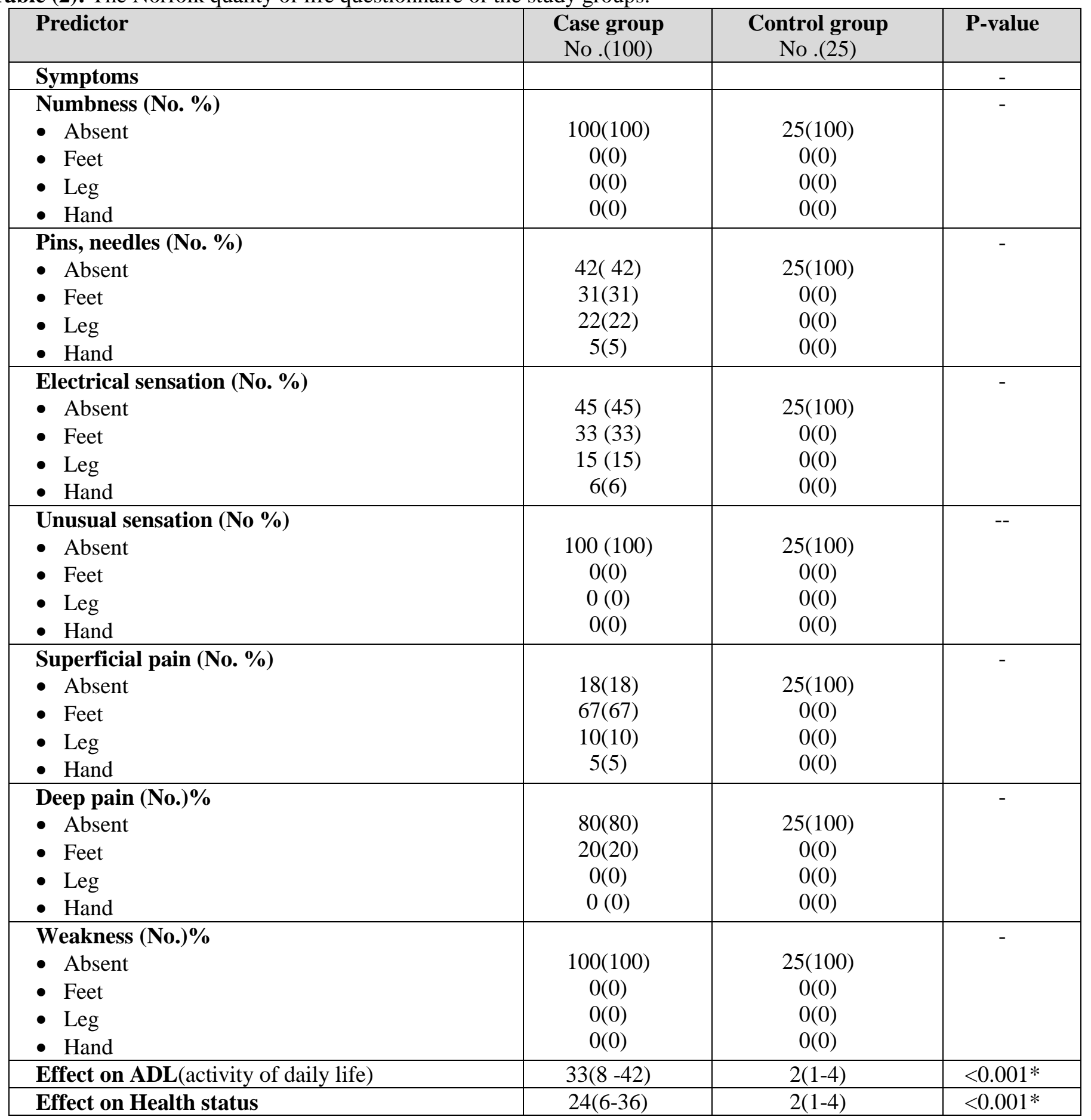

Table (2) showed that Norfolk quality of life questionnaire of diabetic neuropathy showed that the activity of daily life (ADL) and health status were significantly impaired in the case group than the control group $(\mathrm{P}<0.001)$.

Table (3): Simple linear regression analysis of clinical parameters with ADL in patients with diabetic neuropathy.

\begin{tabular}{|l|c|c|}
\hline \multicolumn{1}{|c|}{ Predictor } & B (95\% of Confidence interval) & P-value \\
\hline NSS (neuropathy symptom score) & $6.01(3.15-8.87)$ & 0.001 \\
\hline VAS (visual analogue scale) & $5.09(2.15-8.03)$ & $\leq 0.001$ \\
\hline NDS (neuropathy disability score) & $5.85(3.39-8.30)$ & $\leq 0.001$ \\
\hline Superficial pain & $6.3(0.86: 11.74)$ & 0.024 \\
\hline Duration of DM & $1.33(0.12: 2.55)$ & 0.032 \\
\hline
\end{tabular}




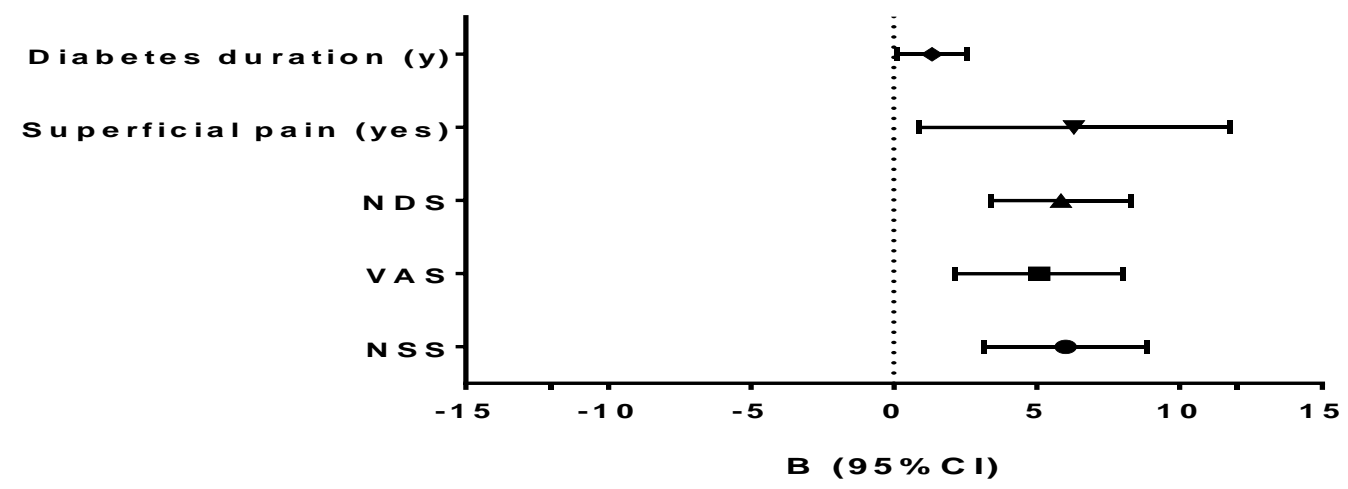

Fig (1): Forest plot showing association with ADL (simple linear regression analysis)

Simple linear regression analysis revealed a significant positive correlation between NSS, VAS, NDS, superficial pain sensation and the duration of DM with ADL $(\mathrm{p} \leq 0.05)$ as shown in table (3) and figure (1).

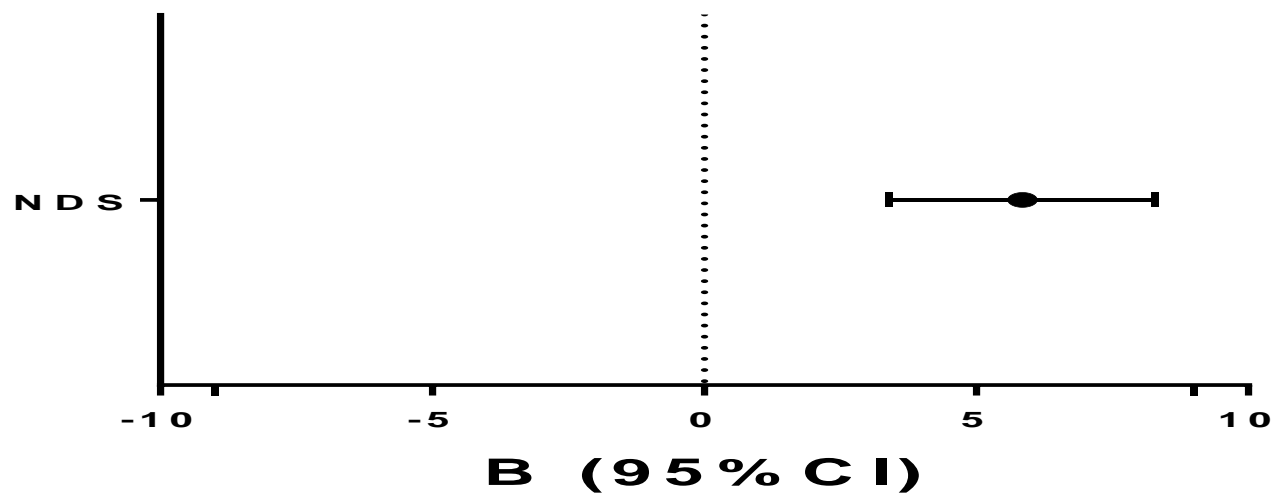

Fig (2): Forest plot showing forward stepwise linear regression analysis using ADL as an outcome (excluded variables: NSS, VAS, Diabetes Duration, Superficial pain)

However, forward stepwise logistic regression analysis of NSS, VAS, NDS, superficial pain and the duration of DM as an independent variables and the effect on ADL (activity of daily life ) as a dependent variable; the NDS was the independent predictor of ADL impairment as shown in figure $(2)(\mathrm{P} \leq 0.05)$.

Table (4): simple linear regression analysis of clinical parameters with the effect on health status in patients with diabetic neuropathy

\begin{tabular}{|l|c|c|}
\hline Predictor & B ( 95\% of Confidence interval) & P-value \\
\hline NSS & $3.91(1.44: 6.38)$ & 0.002 \\
\hline VAS & $3.13(0.61: 5.66)$ & 0.015 \\
\hline NDS & $4.22(2.11: 6.33)$ & $\leq 0.001$ \\
\hline
\end{tabular}

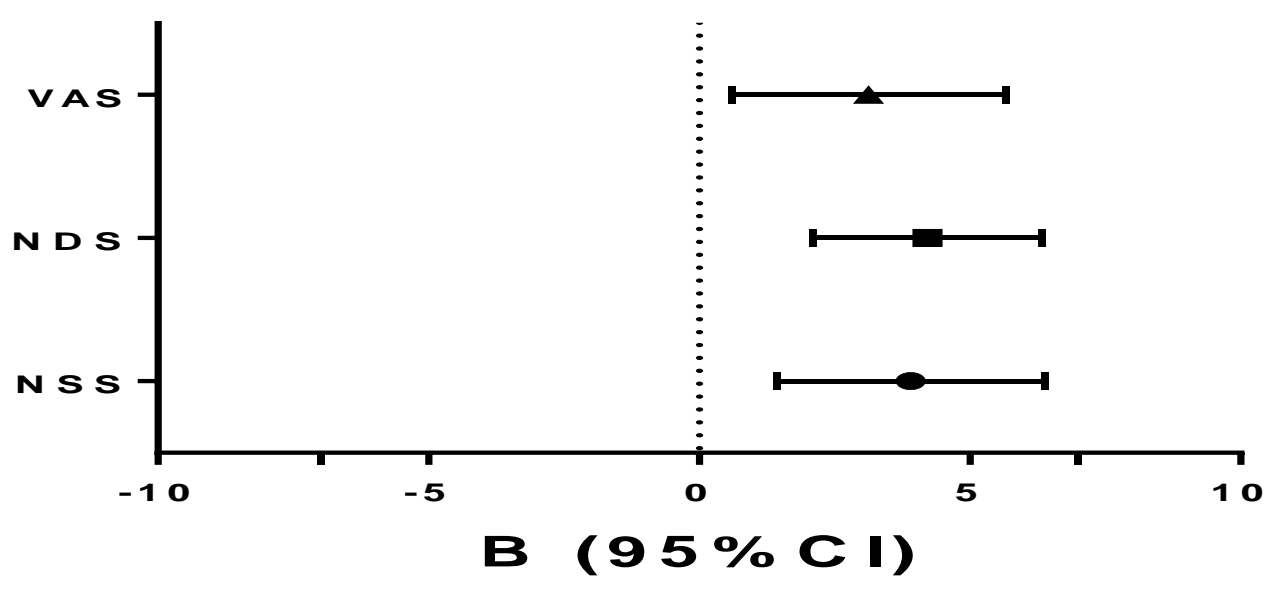

Fig (3): Forest plot showing association with effect on health status (simple linear regression analysis) 


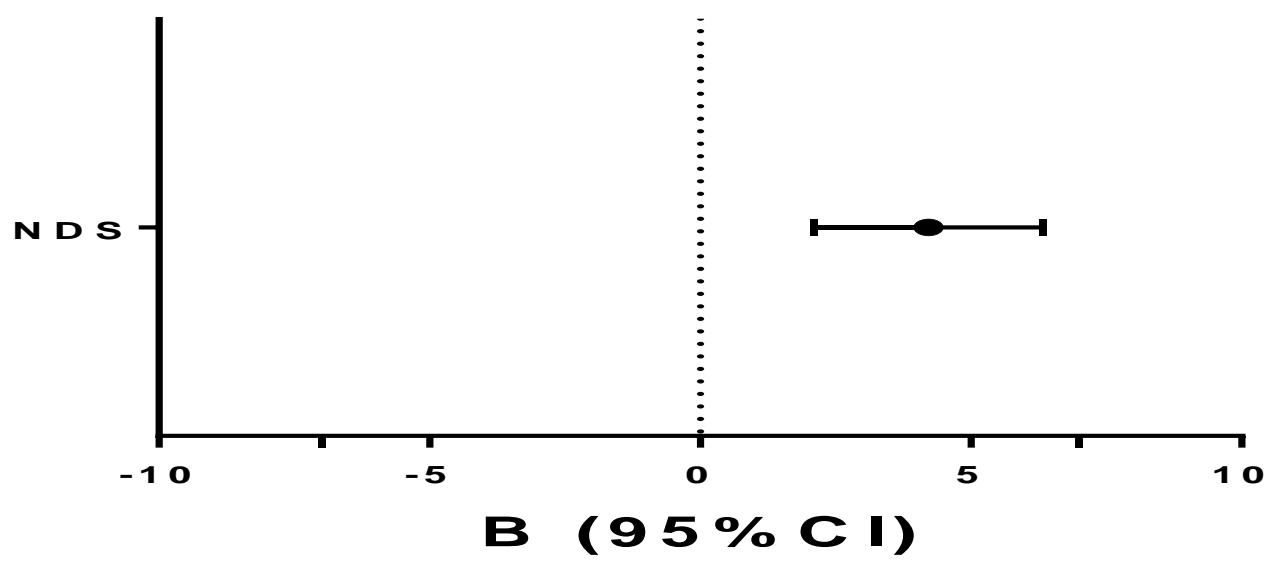

Fig (4): Forest plot showing forward stepwise linear regression analysis using health status as an outcome (excluded variables: NSS, VAS)

Table (4) and figure (3) showed a significant positive correlation between NSS, VAS and NDS with the effect on health status (P-value $\leq 0.05$ ). However, forward stepwise logistic regression analysis of NSS, VAS and NDS as an independent variables and the effect on health status as a dependent variable, the NDS was the independent predictor of the effect on health status as shown in figure (4) $(\mathrm{P} \leq 0.05)$.

Table (5): Association between clinical parameters and HbA1c with axonal neuropathy vs. demyelinating neuropathy in simple logistic regression

\begin{tabular}{|l|c|c|}
\hline \multicolumn{1}{|c|}{ Predictor } & OR (95\% Confidence Interval) & P-value \\
\hline $\begin{array}{l}\text { Medication } \\
\text { Insulin use }\end{array}$ & $3.07(1.21-7.79)$ & 0.019 \\
\hline HbA1C\% & $2.31(1.15-4.69)$ & 0.019 \\
\hline NSS & $3.37(1.46-7.75)$ & 0.005 \\
\hline VAS (visual analogue scale) & $4.61(1.94-10.87)$ & 0.001 \\
\hline NDS & $4.26(2.07-8.77)$ & $\leq 0.001$ \\
\hline $\begin{array}{l}\text { Pins , needles } \\
\text { Yes }\end{array}$ & $5.39(1.69-17.22)$ & 0.004 \\
\hline $\begin{array}{l}\text { Electrical sensation } \\
\text { Yes }\end{array}$ & $4.57(1.55-13.46)$ & 0.006 \\
\hline Duration of DM(y) & $1.62(1.17-2.26)$ & 0.004 \\
\hline
\end{tabular}

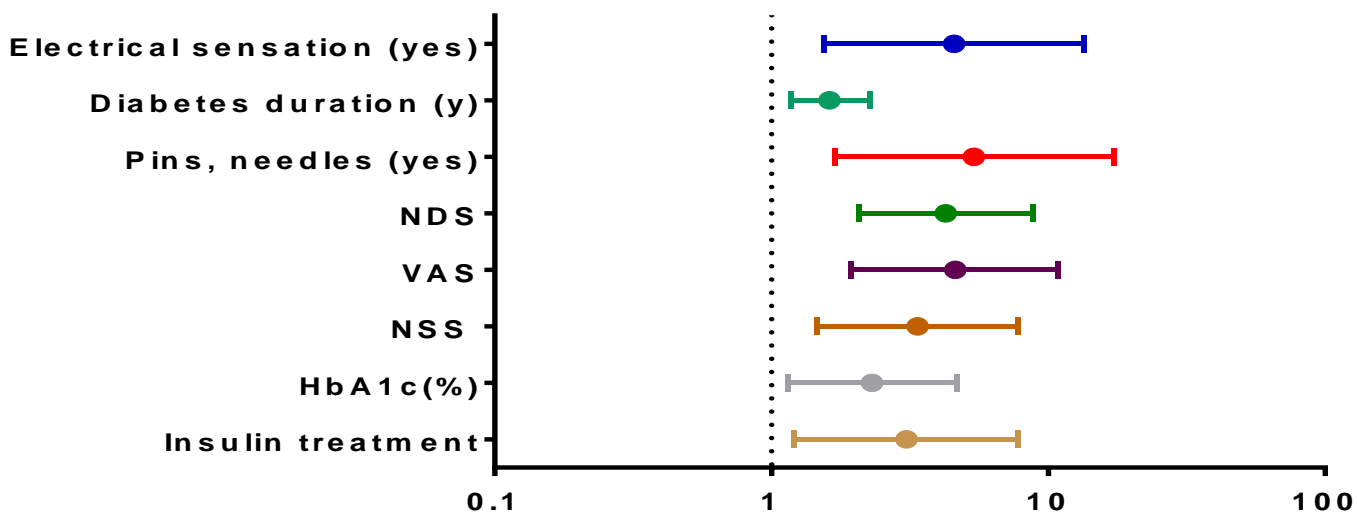

Fig (5): Forest plot showing association between clinical parameters and HbA1c with axonal neuropathy vs. demyelinating in linear regression analysis. 


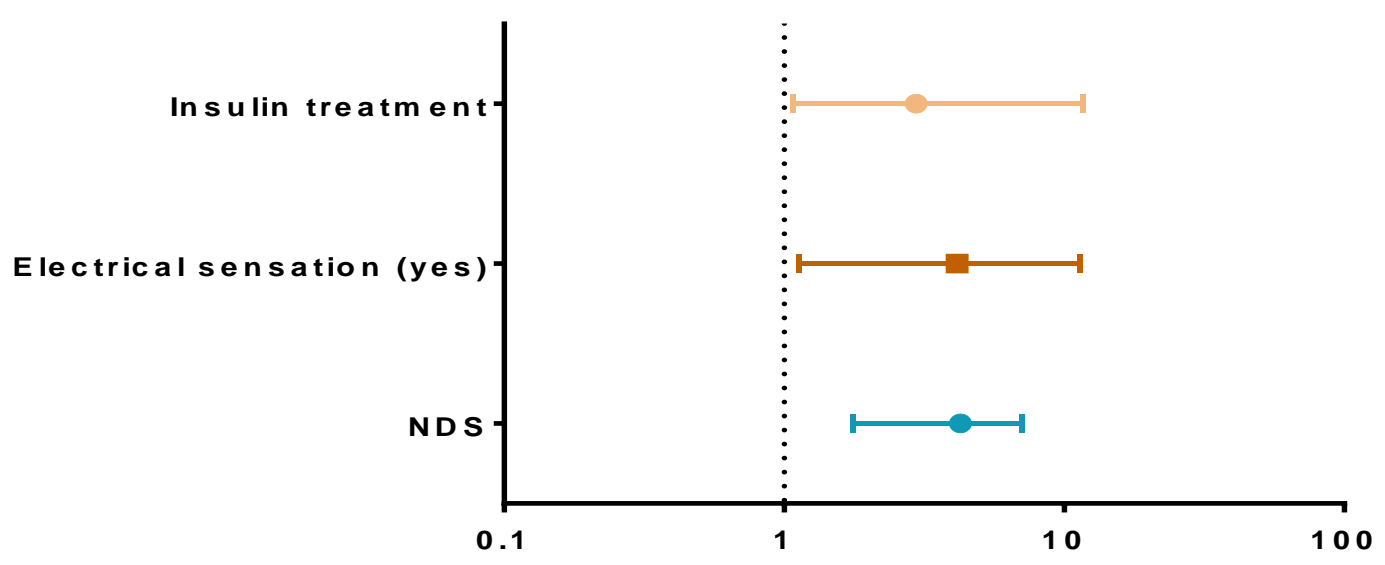

Fig (6): Forest plot showing forward stepwise logistic regression analysis using axonal neuropathy as an outcome (excluded variables: HbA1c, NSS, VAS, duration of DM).

Table (5) and figure (5) showed a significant positive correlation between insulin therapy, HbA1c, electrical sensation, NSS, VAS, NDS and the duration of DM with axonal neuropathy (p 0.05). Forward stepwise logistic regression of NSS, VAS, NDS, HbA1C, insulin therapy and electrical sensation as an independent variables and axonal neuropathy as a dependent variable; the NDS, electrical sensation and insulin therapy were the independent predictors of axonal neuropathy (p 0.05) (Figure 6).

\section{DISCUSSION}

In our study, PDN patients showed an impaired quality of life than those without diabetic neuropathy as regards the effect on activity of daily life and health status.

Regarding age variable, no significant difference was found between the study groups as they were mostly matched in age. However, other studies considered age as a significant risk of lower quality of life particularly in elderly patients (over 65 years old) as they take several medications at the same time for co-morbidities as well as cognitive dysfunction that impair their quality of life $(14,15)$.

In addition, our results revealed non-significant difference in the gender between the study groups. In contrast, other studies detected a significant effect of the gender on quality of life in patients with diabetic neuropathy. Male patients represented a better quality of life for their ability to control diabetes with less liability of depression or anxiety in contrary to female patients that tend to be more expressive of their quality of life impairment. This inconsistency may be attributed to ethnicity and different study methodology ${ }^{(16,17)}$.

Our results of HBA1c exhibited non-significant difference between the two groups. Both groups showed a well-controlled type 2 diabetes mellitus state. In contrast, other researchers found significantly higher HbA1c levels in patients suffering from PDN ${ }^{(18)}$. The impact of intensive glycaemic control on diabetic neuropathy varies in type 1 than type $2 \mathrm{DM}$. In type 1 $\mathrm{DM}$, the intensive glycaemic control could prevent the development of diabetic neuropathy and decrease the risk of nerve conduction dysfunction however in type $2 \mathrm{DM}$, the intensive glycaemic control does not play a major role in the prevention of diabetic neuropathy ${ }^{(\mathbf{1 9})}$. Boussageon et al. ${ }^{(20)}$ attributed this finding to the co-existent other vascular risk factors such as smoking, hypertension, dyslipidaemia, obesity and the duration of DM that impair peripheral nerve integrity.

In our study, the two groups were of matched BMI. Therefore, non-significant difference of BMI between the study groups was found. In contrast, Kolotkin et al. ${ }^{(21)}$ found that overweight and obesity could impair the quality of life due to physical and psychological disabilities.

There was a significant difference between the studied groups in the duration of DM; the longer the duration of DM, the worse was the impact on the quality of life. It was proved to be an independent predictor of axonal neuropathy. This finding agrees with Oguejiofor et al. ${ }^{(22)}$, who considered the duration of DM is a risk factor for diabetic neuropathy.

A multivariate regression analysis of the data of diabetic neuropathy patients showed that the neuropathy disability score (NDS) was the independent predictor of axonal neuropathy and activity of daily life as well as health status impairment. This is in accordance with Abbott et al. ${ }^{(12)}$ who considered the NDS as a good reliable tool to predict diabetic foot ulcers, which is the end result of neuropathy.

The Norfolk quality of life questionnaire (QOL$\mathrm{DN}$ ) in our research showed that people with diabetic neuropathy have a worse quality of life than those without diabetic neuropathy regarding the activity of daily life and health status impairment. This agrees with Vinik et al. ${ }^{(23)}$, who showed that the QOL-DN accurately 
and effectively identifies and specifies diabetic peripheral neuropathy with a reliable diagnosis of diabetic neuropathy. Also Van Acker et al. ${ }^{(24)}$ found a significant disruption of both the physical and psychological health components of quality of life in diabetic patients.

Our strength points were the close matching between the two groups to avoid co-founders such as age, body weight and HBA1c and the use of multiple tools in the diagnosis and assessment of PDN as well as in discrimination of its impact on various quality of life aspects.

The limitations in our study were the conduction of the study in the same setting in a single centre upon single ethnicity. However, the tools for diagnosis of DPN vary in their sensitivity, specificity, and accuracy, particularly in different ethnicities. More studies with larger scale of multi-ethnicities are warranted in the future.

\section{CONCLUSION}

Egyptian patients with type 2 diabetes mellitus and PDN are more vulnerable to impaired quality of life particularly in the activity of daily life and health status. The neuropathy disability score (NDS) was the independent predictor of axonal neuropathy, activity of daily life and health status deterioration.

Acknowledgment: not applicable.

Conflict of interest: nil.

\section{REFERENCES}

1. Casellini C, Vinik A (2007): Clinical Manifestations and Current Treatment Options For Diabetic Neuropathies. Endocrine Practice, 13: 550-566.

2. Ziegler D (2010): Can Diabetic Polyneuropathy Be Successfully Treated? MMW Fortschritte Der Medizin, 152: 64-68.

3. Tesfaye S (2009): Advances In The Management Of Diabetic Peripheral Neuropathy. Current Opinion in Supportive and Palliative Care, 3: 136-143.

4. Uzar E, Alp H, Cevik M et al. (2012): Ellagic Acid Attenuates Oxidative Stress On Brain And Sciatic Nerve And Improves Histopathology Of Brain In Streptozotocin-Induced Diabetic Rats. Neurological Sciences, 33: 567-574.

5. Sugimoto K, Yasujima M, Yagihashi S (2008): Role of Advanced Glycation End Products In Diabetic Neuropathy. Current Pharmaceutical Design, 14: 953- 961.

6. Shimoshige Y, Enomoto R, Aoki T et al. (2010): The Involvement of Aldose Reductase In Alterations To Neurotrophin Receptors And Neuronal Cytoskeletal Protein Mrna Levels In The Dorsal Root Ganglion Of StreptozotocinInduced Diabetic Rats. Biological and Pharmaceutical Bulletin, 33: 67-71.

7. Hoffman D, Sadosky A, Alvir J (2009): Cross-National Burden of Painful Diabetic Peripheral Neuropathy In Asia, Latin America, And The Middle East. Pain Practice, 9: 35-42.

8. Ramos K, Jiang Y, Svensson C et al. (2007): Pathogenesis of Spinally Mediated Hyperalgesia In Diabetes. Diabetes, 56: 1569-1576.
9. Rijken P, Dekker J, Lankhorst G et al. (1999): Podiatric Care for Diabetic Patients With Foot Problems: An Observational Study. International Journal of Rehabilitation Research, 22: 181- 188.

10. Vinik E, Hayes R, Oglesby A et al. (2005): The Development and Validation of the Norfolk Qol- Dn, a New Measure of Patients' Perception of the Effects of Diabetes and Diabetic Neuropathy. Diabetes Technology \& Therapeutics, 7: 497-508.

11. Young M, Boulton A, Macleod A et al. (1993): A Multicentre Study of the Prevalence of Diabetic Peripheral Neuropathy in the United Kingdom Hospital Clinic Population. Diabetologia, 36: $150-154$.

12. Abbott C, Carrington A, Ashe $\mathrm{H}$ et al. 2(002): The NorthWest Diabetes Foot Care Study: Incidence Of, And Risk Factors For, New Diabetic Foot Ulceration in a CommunityBased Patient Cohort. Diabetic Medicine, 19: 377-384.

13. Gillian Z H, Maurizio M, Roberta C (2016): How to analyze the Visual Analogue Scale: Myths, truths and clinical relevance. Scand J Pain, 13: 67-75.

14. Papelbaum M, Lemos H, Duchesne $M$ et al. (2010): The Association between Quality Of Life, Depressive Symptoms and Glycemic Control in a Group of Type 2 Diabetes Patients. Diabetes Research and Clinical Practice, 89: 227-230.

15. Spasić A, Radovanović R, Đorđević A et al. (2014): Quality Of Life in Type 2 Diabetic Patients. Acta Facultatis Medicae Naissensis, 31: 193-200.

16. Imayama I, Plotnikoff R, Courneya K et al. (2011): Determinants of Quality Of Life in Adults with Type 1 and Type 2 Diabetes. Health and Quality of Life Outcomes, 9: 115118.

17. Varghese R, Salini R, Abraham P et al. (2007): Determinants of the Quality Of Life among Diabetic Subjects in Kerala, India. Diabetes \& Metabolic Syndrome: Clinical Research \& Reviews, 1: 173-9.

18. Smith A, Singleton J (2013): Obesity and Hyperlipidemia are Risk Factors for Early Diabetic Neuropathy. Journal of Diabetes and Its Complications, 27: 436-442.

19. Brian C, Hsinlin C, Catherine $L$ et al. (2012): Diabetic neuropathy: Clinical manifestations and current treatments. Lancet Neurol., 11 (6): 521-534.

20. Boussageon R, Bejan-Angoulvant T, Saadatian-Elahi M et al. (2011): Effect Of Intensive Glucose Lowering Treatment On All-Cause Mortality, Cardiovascular Death, And Microvascular Events In Type 2 Diabetes: Meta-Analysis Of Randomised Controlled Trials. BMJ., 26: 343-48.

21. Kolotkin R, Crosby R, Williams G (2002): Health-Related Quality Of Life Varies Among Obese Subgroups. Obesity Research, 10 (8): 748-756.

22. Oguejiofor O, Odenigbo C, Oguejiofor C (2010): Evaluation Of The Effect Of Duration Of Diabetes Mellitus On Peripheral Neuropathy Using The United Kingdom Screening Test Scoring System, Bio-Thesiometry And Aesthesiometry. Nigerian Journal of Clinical Practice, 13 (3): 240-7

23. Vinik E, Paulson J, Ford-Molvik S et al. (2008): GermanTranslated Norfolk Quality Of Life (Qol-Dn) Identifies The Same Factors As The English Version Of The Tool And Discriminates Different Levels Of Neuropathy Severity. Journal of Diabetes Science and Technology, 2 (6): 1075-1086.

24. Van Acker K, Bouhassira D, De Bacquer D et al. (2009): Prevalence And Impact On Quality Of Life Of Peripheral Neuropathy With Or Without Neuropathic Pain In Type 1 And Type 2 Diabetic Patients Attending Hospital Outpatients Clinics. Diabetes \& Metabolism, 35: 206-213. 